\title{
The cardioprotective potential of valsartan in myocardial ischaemia reperfusion injury
}

\author{
NAJAH. R. HADI, FHADIL. G. AL-AMRAN, YASMEEM. A. HUSSIEN, ISRAA. K. AL-YASIRI, \\ MAHAMED AL-TURFY
}

University of Kufa, Najaf, Iraq

\begin{abstract}
Background: Ischaemia/reperfusion injury describes the experimentally and clinically prevalent finding that tissue ischaemia with inadequate oxygen followed by successful reperfusion initiates a wide and complex array of inflammatory responses that may aggravate local injury as well as induce impairment of remote organ function by mechanisms that involve oxidative stress, inflammation, and apoptosis.

Objective: This study was undertaken to investigate the potential role of valsartan angiotensin receptor blocker-1 (ARB-1) in the amelioration of myocardial ischaemia/reperfusion injury induced by ligation of coronary artery in a rat model.

Material and methods: Adult male Albino rats were randomised into four equal groups (seven rats in each group). In group 1 (sham group) the rats underwent the same anaesthetic and surgical procedure as the control group except for ligation of the left anterior descending (LAD) coronary artery; group 2 (control group) rats were subjected to regional ischaemia for 25 minutes by ligation of LAD coronary artery and reperfusion for 2 hours; group 3 (control vehicle group) rats received (normal saline) vehicle of valsartan via IP injection and were subjected to regional ischaemia for 25 minutes by ligation of LAD coronary artery and reperfusion for two hours; group 4 (valsartan treated group) rats were pretreated with valsartan $10 \mathrm{mg} / \mathrm{kg}$ IP 30 minutes before ligation of LAD coronary artery. At the end of the experiment, blood samples were taken by direct cardiac puncture for the measurement of plasma levels of troponin $T(c T n T)$ and serum levels for both malondialdehyde MDA and glutathione GSH. After blood sampling, the heart was removed and divided into two parts; the apex was used for histopathological examination, and the remaining part was used for the measurement of cardiac tissue levels of tumour necrosis factor $\alpha$ $(T N F-\alpha)$, interleukin 6 (IL-6), interleukin 10 (IL-10), cysteine aspartic acid-protease 3 (caspase-3), and $B C L 2$-associated X protein (BAX), after removal of the remaining blood clots and aorta.

Results: In the active control group, as compared with the sham group, the results revealed that the myocardial tissue levels of inflammatory cytokines $T N F-\alpha, I L-6, I L-10$, caspase-3, and BAX, and the plasma level of cTnT and serum level of malondialdehyde MDA were significantly increased $(p<0.001)$, while the serum level of glutathione GSH was significantly decreased $(p<0.001)$. Regarding the histopathological part of the study, all rats in the active control group showed a significant cardiac tissue injury $(p<0.001)$ compared with the sham group. Valsartan significantly counteracted $(p<0.001)$ the increase in the myocardial tissue levels of TNF- $\alpha, I L-6$, caspase-3, and BAX; additionally, it counteracted the increase in plasma level of $c T n T$ and serum level of malondialdehyde MDA, while valsartan produced highly significant elevation $(p<0.001)$ in the cardiac tissue level of $I L-10$ and serum level of glutathione GSH and significantly reduced $(p<0.001)$ the cardiac tissue injury in the valsartan pretreated rats.

Conclusions: The results of the present study reveal that valsartan ameliorates myocardial ischaemia reperfusion injury in rats by interfering with inflammatory reactions and apoptosis that are induced by ischaemia reperfusion injury.
\end{abstract}

Key words: ischaemia reperfusion injury, valsartan.

(Centr Eur J Immunol 2015; 40 (2): 159-166)

\section{Introduction}

Although restoration of blood flow to an ischaemic organ is essential to prevent irreversible cellular injury, reperfusion may enhance tissue injury in excess of that produced by ischaemia alone [1]. Ischaemia contributes to the pathophysiology of many conditions, including myocardial infarction, peripheral vascular insufficiency, stroke, and hypovolemic shock. The major mecha-

Correspondence: Israa. K. Al-Yasiri, PhD, University of Kufa, Najaf, Iraq, e-mail: israayasiri@yahoo.com 
nisms of ischaemic reperfusion injury are oxidative stress (mainly oxygen-free radicals), apoptosis, neutrophil-endothelium interactions, and hypercontracture (i.e. myocyte $\mathrm{Ca}^{2+}$ over loading), endothelial cell activation with microvascular dysfunction, and altered myocardial metabolism. Ischaemic reperfusion injury prompts a release of cytokines and other proinflammatory mediators that activate both the neutrophils and the coronary vascular endothelium. Adaptive cellular responses activate the innate immune system with its toll-like receptors (TLRs) and the complement system as well as the adaptive immune system.

Valsartan is a potent, non-peptide tetrazole derivative and selectively inhibits angiotensin II receptor type 1 [2]. Selective angiotensin receptor blocker-1 (ARB-1) exerts a variety of pleiotropic effects, including anti-oxidative, anti-apoptotic, and anti-inflammatory effects [3]. Navalkar et al. found that the cardioprotective effects of valsartan ARB-1 are related to mechanisms beyond blood pressure lowering, anti-inflammation, anti-atherosclerosis, and target organ protection [4].

Valsartan inhibited Toll-like receptor 4 (TLR-4) and nuclear factor-kappa B (NF-кB) expressions concomitant with an improvement in myocardial injury, such as smaller infarct size, reduced release of myocardial enzymes, and proinflammatory mediators. Varagic et al. suggested that valsartan plays an essential role in the protective effects on myocardial ischemic reperfusion injury, and the possible protection mechanism is due to its anti-inflammation function via TLR-4/NF- $\kappa \mathrm{B}$ signalling pathway [5].

\section{Material and methods}

After the approval from the Institutional Animal Care and Use Committee (IACUC) in Kufa University and submission of the required applications, a total of 28 adult male albino rats weighting (200-250 g) were purchased from the Animal Resource Centre of the National Centre for Drug Control and Research. They were housed in the animal house of Kufa University/College of Medicine in a temperature-controlled $\left(25 \pm 1^{\circ} \mathrm{C}\right)$ room (humidity was kept at 60-65\%) with alternating 12 hours light/12 hours dark cycle and were allowed free access to water and chow diet until the start of experiments.

\section{Study design}

After two weeks of acclimatisation 28 rats were randomised into four groups, seven rats in each group, as follows:

in Group I (Sham): rats underwent to the same anaesthetic and surgical procedure but without ligation of the left anterior descending artery (LAD);

in Group II (control group): rats underwent myocardial ischaemia for 30 minutes by ligation of LAD and reperfusion for two hours; in Group III (vehicle group): rats pretreated with normal saline (vehicle for valsartan) were given intraperitoneal injection 30 minutes before ligation of LAD and reperfusion for two hours;

in Group IV (valsartan-pretreated I/R group): rats pretreated with valsartan $10 \mathrm{mg} / \mathrm{kg}$ dissolved in saline solution [6], intraperitoneal injection 30 minutes before ligation of LAD and reperfusion for two hours. The valsartan was prepared immediately before injection.

\section{Surgical ligation of anterior descending artery}

All rats were anesthetised by intraperitoneal (IP) injection of $100 \mathrm{mg} / \mathrm{kg}$ ketamine and $5 \mathrm{mg} / \mathrm{kg}$ xylazine [7]. After intubation of the trachea by a $20 \mathrm{fg}$ cannula the endotracheal tube was connected tightly to the ventilation machine. The ventilation rate was fixed at $120-135$ breaths/minute with a tidal volume of $20 \mathrm{ml} / \mathrm{kg}$ body weight, with $100 \%$ oxygen. The intercostal muscle layer was gradually cut with micro fine scissors. After that the pericardium was opened so the left ventricle was visible, and the LAD was ligated with an 8-0 Prolene suture. The chest wall was closed and at the end of reperfusion time the animal was re-anesthetised by IP a mixture of $100 \mathrm{mg} / \mathrm{kg}$ ketamine and $5 \mathrm{mg} / \mathrm{kg}$ xylazine, and the chest was re-opened, then the right ventricle was punctured with a syringe needle so that about $3 \mathrm{ml}$ of blood was aspirated for later blood analysis. After that, the heart was isolated and divided into two pieces; the apical part was used for histological examination, and the basal was used for measuring the tissue parameters.

\section{Preparation of samples \\ Blood sampling for measurement of plasma CTNT, serum MDA, and serum reduced GSH}

At the end of the experiment, about 2-3 $\mathrm{ml}$ of blood was collected by disposable syringe from the heart of each rat via cardiac puncture. The first half was immediately placed in a tube containing disodium EDTA $(22 \mathrm{mg} / \mathrm{ml})$ as an anticoagulant and mixed thoroughly then centrifuged at $3000 \mathrm{rpm}$ for 15 minutes. Then the supernatant was used for determination of the plasma cTnT cardiac troponin $\mathrm{T}$ level. The remaining blood was allowed to clot in an ordinary tube at $37^{\circ} \mathrm{C}$ and centrifuged at $3000 \mathrm{rpm}$ for 15 minutes, and then the supernatant was used for the determination of serum malondialdehyde MDA and glutathione GSH levels.

\section{Tissue preparation for $T N F-\alpha, I L-6, I L-10$, caspase 3, and BAX measurements}

The heart of each rat was excised at the end of the reperfusion time, washed with ice-cold saline to exclude clots, any remaining parts of the atria were removed, and then homogenisation for the cardiac tissue was done with a high-intensity ultrasonic liquid processor in $1: 10(\mathrm{w} / \mathrm{v})$ phosphate buffered saline that contained $1 \%$ Triton X-100 and a prote- 
ase inhibitor cocktail [8]. Then tissue homogenate was centrifuged at $14,000 \mathrm{rpm}$ for 20 minutes at $4^{\circ} \mathrm{C}$. After this step, the collection of the tissue homogenate supernatant was done in order to detect the levels of TNF- $\alpha$, IL-6, IL-10, caspase 3, and BAX by the ELISA technique with a commercially available ELISA kit (literature of kit by Biotangusa, USA) according to the manufacturer's instructions.

\section{Tissue preparation for histopathology}

The myocardial tissue that was reserved for histopathological study was fixed in $10 \%$ formalin and embedded in a block of paraffin. The 5- $\mu \mathrm{m}$ sections that were cut from each block were stained by haematoxylin and eosin (H\&E) after fixation. Damage scores were evaluated according to the following morphological criteria that have been used to evaluate the histopathological damage [9] as follow:

score 0 , no damage;

score 1 (mild), interstitial oedema and focal necrosis; score 2 (moderate), diffuse myocardial cell swelling and necrosis;

score 3 (severe), necrosis with the presence of contraction bands, neutrophil infiltration, and the capillaries were compressed;

score 4 (highly severe), widespread necrosis with the presence of contraction bands, neutrophil infiltration, capillaries compressing, and haemorrhage.

\section{Statistical analysis}

Data were expressed as mean \pm SEM. Expert statistical advice was considered for data analysis, which was aided by computer. Statistical analysis was done using SPSS version 20.0 software (Statistical Package for Social Science).

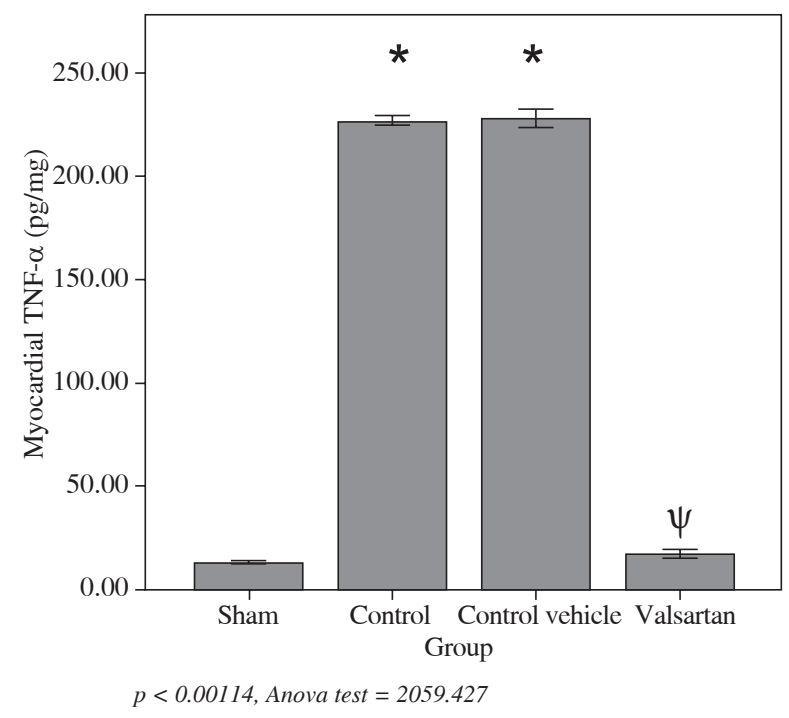

Fig. 1. Mean of myocardial TNF- $\alpha$ level (pg/mg) *vs. sham group, $\psi$ vs. control vehicle group
ANOVA (analysis of variance) was used for measurement (numerical data). Mann-Whitney test was used for myocardial damage score. A $p$ value $<0.05$ was regarded as significant.

\section{Results}

\section{Effect on pro-inflammatory cytokines (TNF- $\alpha$ )}

The results revealed a significant increase $(p<0.001)$ in TNF- $\alpha$ tissue levels in the active control group compared with the sham group (mean \pm SD: $227.7571 \pm 12.18522$ ), while in the valsartan-pretreated group, a highly significant reduction was seen $(p<0.001)$ in the TNF- $\alpha$ tissue levels compared with the active control group and vehicle group $(17.1900 \pm 5.49024)$, as shown in Fig. 1 .

\section{Effect on pro-inflammatory cytokines (IL-6)}

The results revealed a significant increase $(p<0.001)$ in IL-6 tissue levels in the active control group compared with the sham group $(202.7143 \pm 15.93439)$, while in the valsartan-pretreated group a highly significant reduction was seen $(p<0.001)$ in the IL-6 tissue levels compared with the active control group and vehicle group (17.2857 \pm 5.99206 ), as shown in Fig. 2 .

\section{Effect on anti-inflammatory cytokine (IL-10)}

The results revealed a significant increase $(p<0.001)$ in IL-10 tissue level in the active control group compared with the sham group $(51.5457 \pm 7.73942)$, while in the valsartan-pretreated group, a significant elevation was seen $(p<0.001)$ in the IL-10 tissue level compared with all oth-

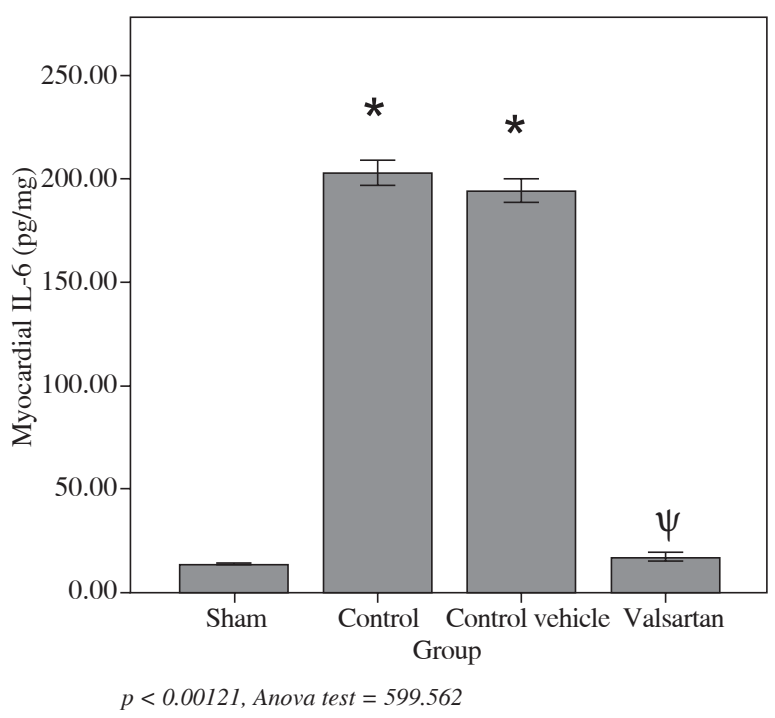

Fig. 2. Mean of myocardial IL-6 level (pg/mg) vs. sham group, $\psi$ vs. control vehicle group 
er groups (sham group, active control group, and vehicle group) (189.1414 \pm 10.07340$)$, as shown in Fig. 3 .

\section{Effect on apoptotic markers (caspase-3)}

The results revealed a significant increase $(p<0.001)$ in caspase- 3 tissue levels in the active control group compared with the sham group $(3.4043 \pm 0.13831)$, while in the valsartan-pretreated group, a highly significant reduction was seen $(p<0.001)$ in the caspase- 3 tissue levels compared with the active control group and vehicle group (1.1586 \pm 0.13069$)$, as shown in Fig. 4.

\section{Effect on apoptotic markers (BAX)}

The results revealed a significant increase $(p<0.001)$ in BAX tissue levels in the active control group compared with the sham group $(4.1014 \pm 0.14519)$, while in the valsartan-pretreated group, a highly significant reduction was seen $(p<0.001)$ in the BAX tissue levels compared

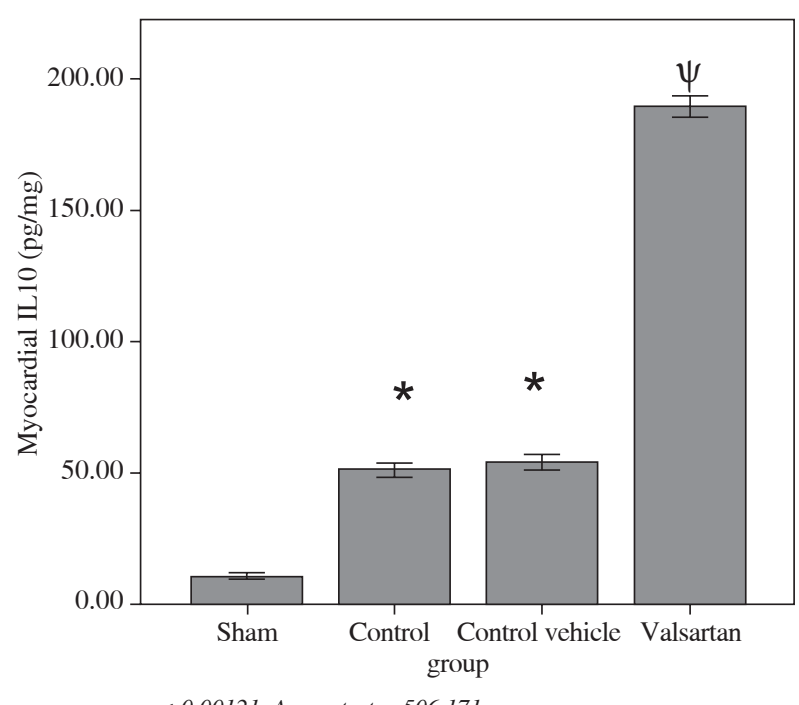

$p<0.00121$, Anova test $=506.171$

Fig. 3. Mean of myocardial IL-10 level (pg/mg) *vs. sham group, $\psi$ vs. control vehicle group

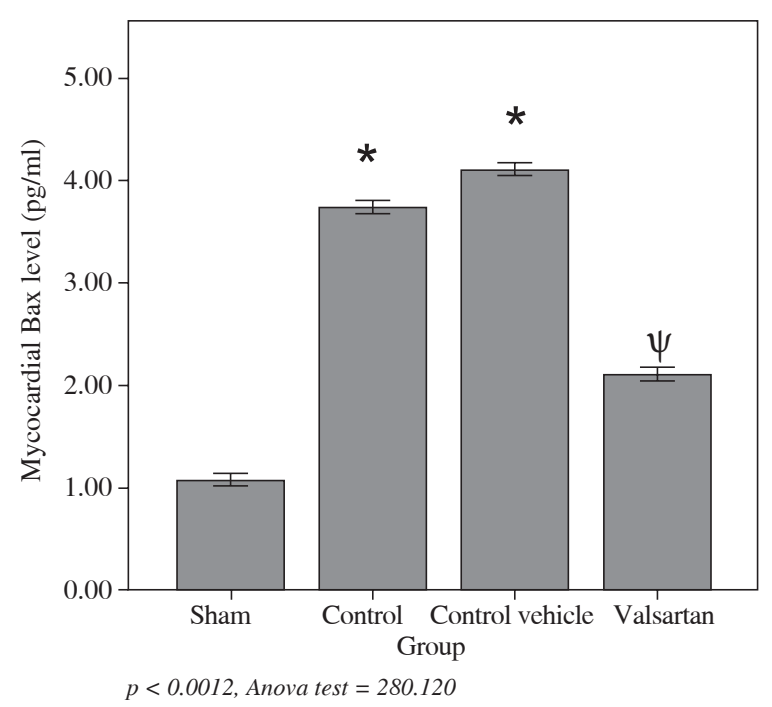

Fig. 5. Mean of myocardial BAX level (pg/mg) *vs. sham group, $\psi$ vs. control vehicle group

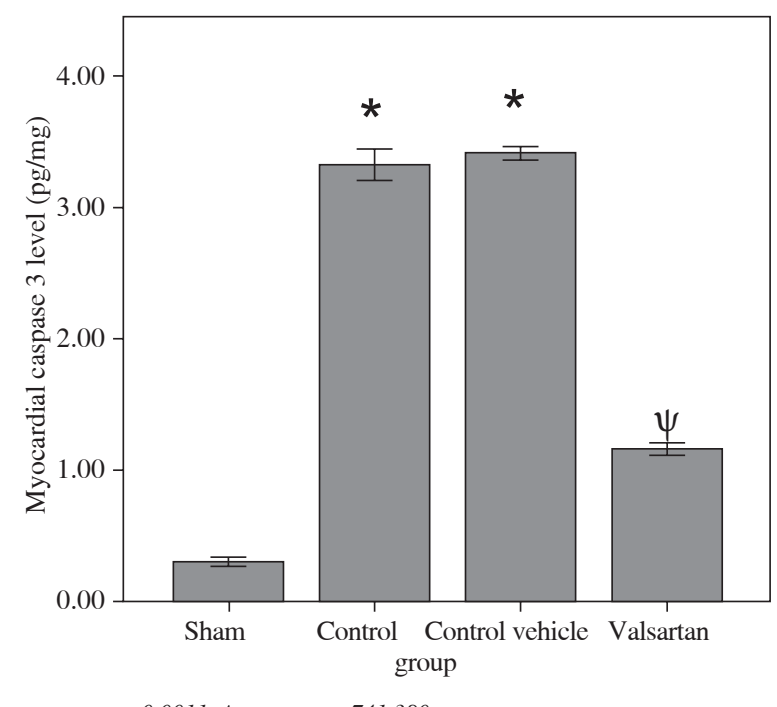

$p<0.0011$, Anova test $=741.380$

Fig. 4. Mean of myocardial caspase 3 level (pg/mg) * vs. sham group, $\psi$ vs. control vehicle group

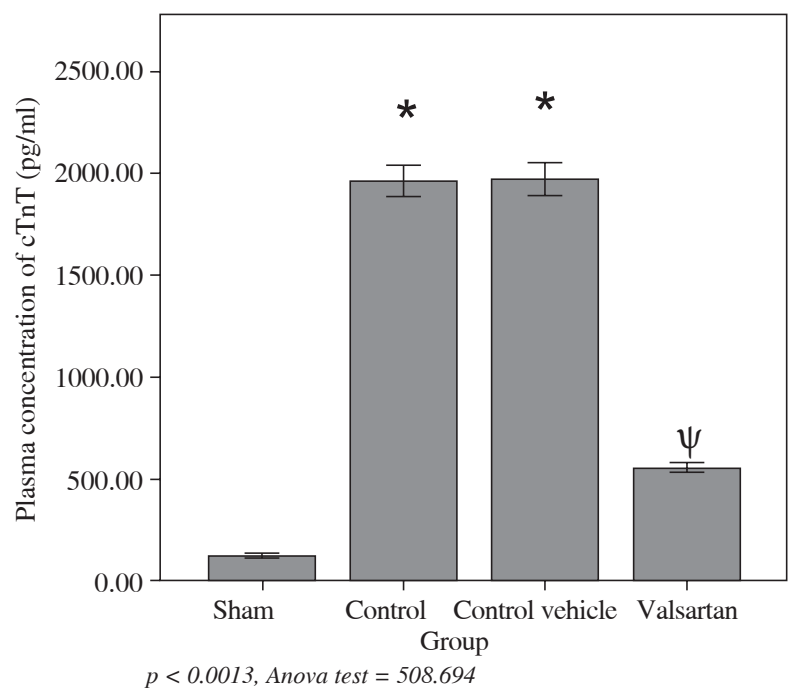

Fig. 6. Mean of myocardial CTnT level (pg/ml) *vs. sham group, $\psi$ vs. control vehicle group 


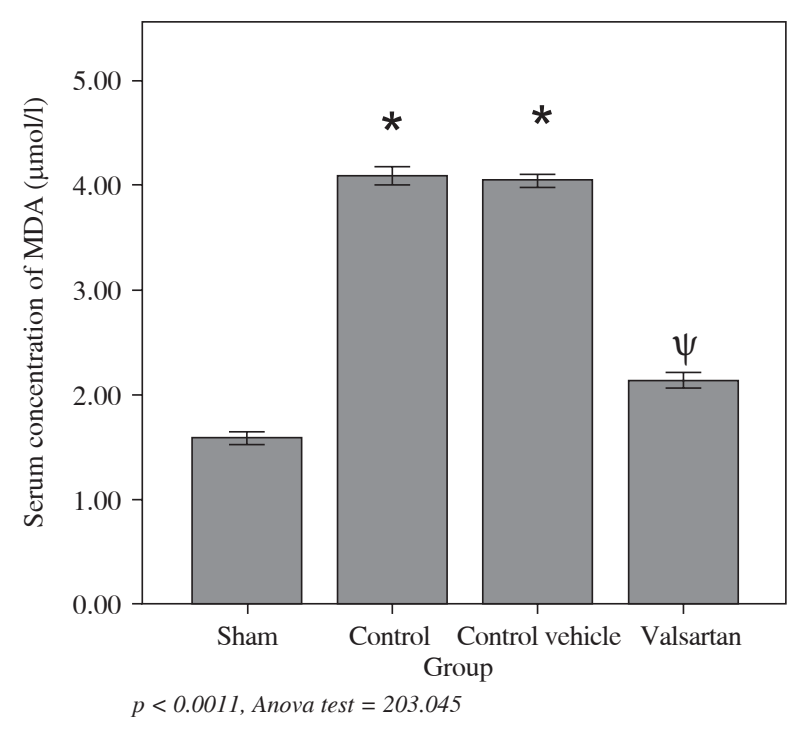

Fig. 7. Serum concentration of MDA $(\mu \mathrm{mol} / \mathrm{l})$ *vs. sham group, $\psi$ vs. control vehicle group

with the active control group and vehicle group (2.1100 \pm 0.16961 ), as shown in Fig. 5 .

\section{Effect of troponin $\mathbf{T}$ (cTnT) on plasma level}

The results revealed a significant increase $(p<0.001)$ in cTnT plasma level in the active control group compared with the sham group $(1967.5714 \pm 207.52017)$, while in the valsartan-pretreated group, a highly significant reduction $(p<0.001)$ in the cTnT plasma level compared with the active control group and vehicle group was revealed (551.2857 \pm 64.53607$)$, as shown in Fig. 6 .

\section{Effect of oxidative stress marker malondialdehyde (MDA) on the serum level}

The results revealed a significant increase $(p<0.001)$ in the serum level of MDA in the active control group compared with the sham group (4.0514 \pm 0.11978$)$, while in the valsartan-pretreated group, a significant reduction $(p<0.001)$ in MDA serum level was seen compared with the active control group and vehicle group (4.0514 \pm 0.11978 ), as shown in Fig. 7 .

\section{Effect of oxidative stress marker glutathione (GSH) on the serum level}

The results revealed a significant decrease $(p<0.001)$ in the serum level of GSH in the active control group compared with the sham group $(28.1429 \pm 2.26779)$, while in the valsartan-pretreated group, a significant increase $(p<0.001)$ in GSH serum level as compared with the active control group and vehicle group was seen (43.5714 \pm 3.77964 ), as shown in and Fig. 8 .

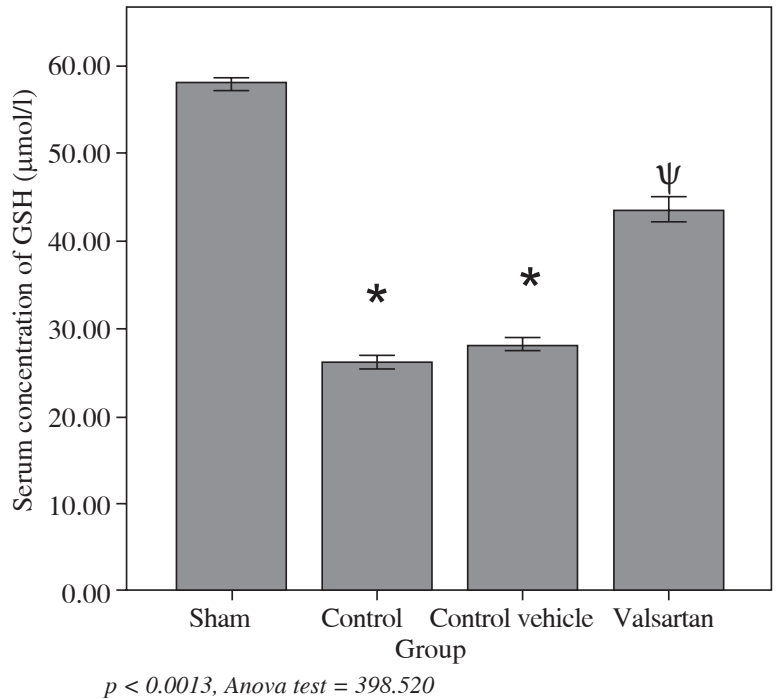

Fig. 8. Serum concentration of GSH ( $\mu \mathrm{mol} / \mathrm{l})$

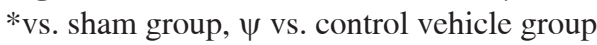

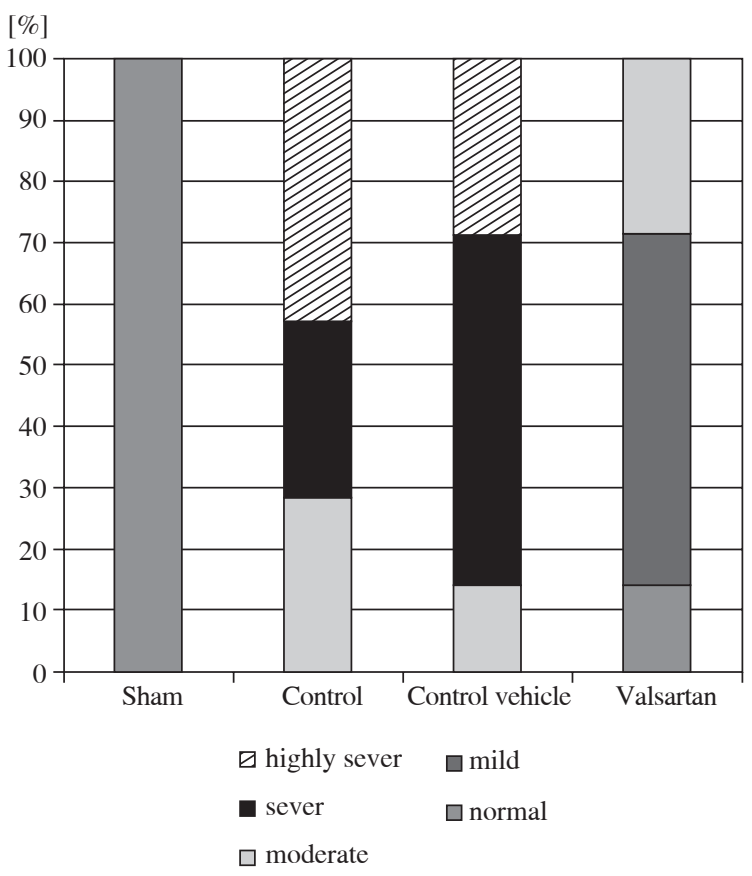

Fig. 9. Component bar chart of the relative frequency of different histopathology grading of abnormal heart changes among the four experimental groups

\section{Histopathological findings}

Histopathological study was carried out by an independent pathologist who was unaware of the treatment given. A cross-section of heart tissue obtained from the sham group showed normal cardiac structure (score 0); 


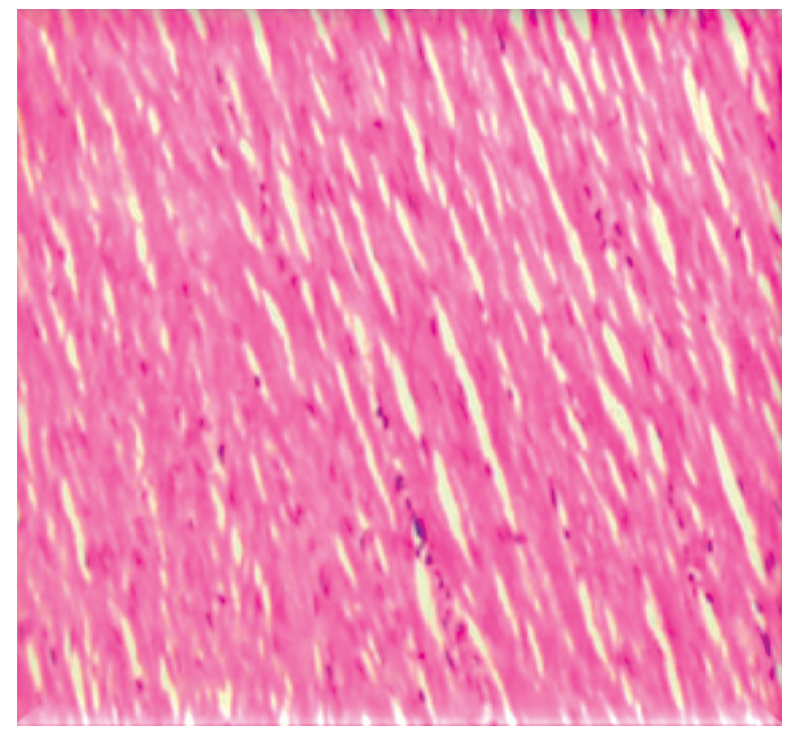

Fig. 10. Section from the cardiac tissue from the sham group clarifying the normal architecture. This section was stained with H\&E (40×)

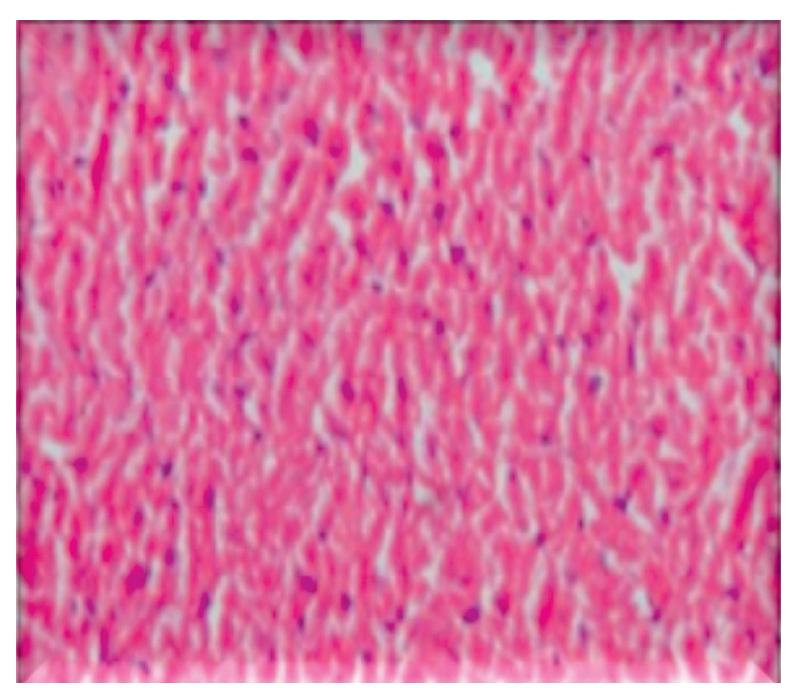

Fig. 12. Section of cardiac tissue for the control vehicle group showed hemorrhage, heavy neutrophil infiltration, oedema and necrosis. The section was stained with H\&E (40×)

no interstitial oedema, no diffuse myocardial cell swelling and necrosis, no neutrophils infiltration, no haemorrhage, no capillary compression, and no evidence of apoptosis. There was a statistically significant difference between the control group and the sham group $(p<0.05)$, and the total severity scores of control group showed that $28.6 \%$ of the group had severe cardiac injury (score 3 ), $28.6 \%$ had moderate cardiac injury (score 2 ), and $42.8 \%$ had a highly severe cardiac injury (score 4 ). There was a statistically

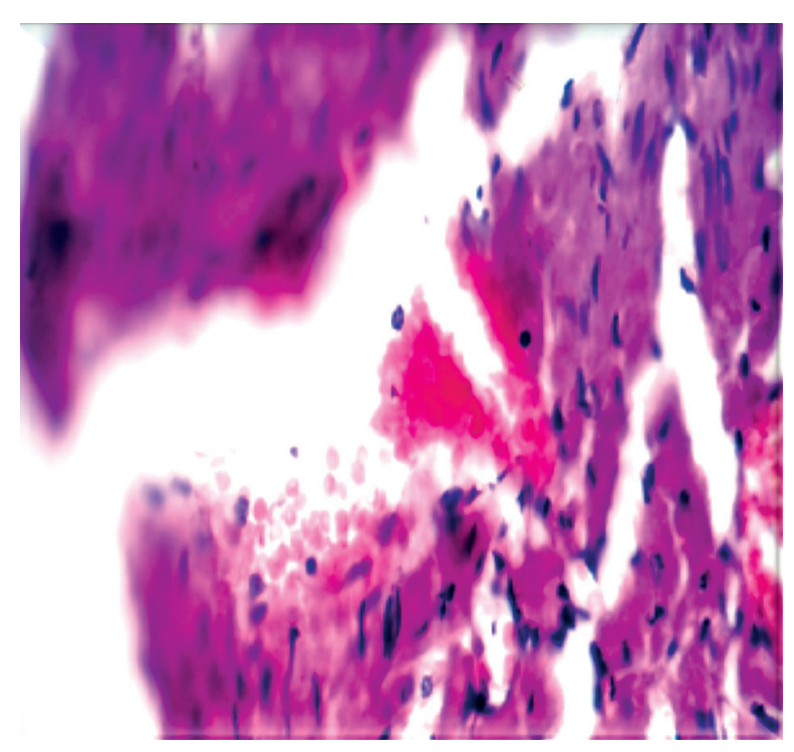

Fig. 11. Section for the cardiac tissue from the active control group showing sever hemorrhage, interstitial oedema, and neutrophil infiltration. The section was stained with $\mathrm{H} \& \mathrm{E}(40 \times)$

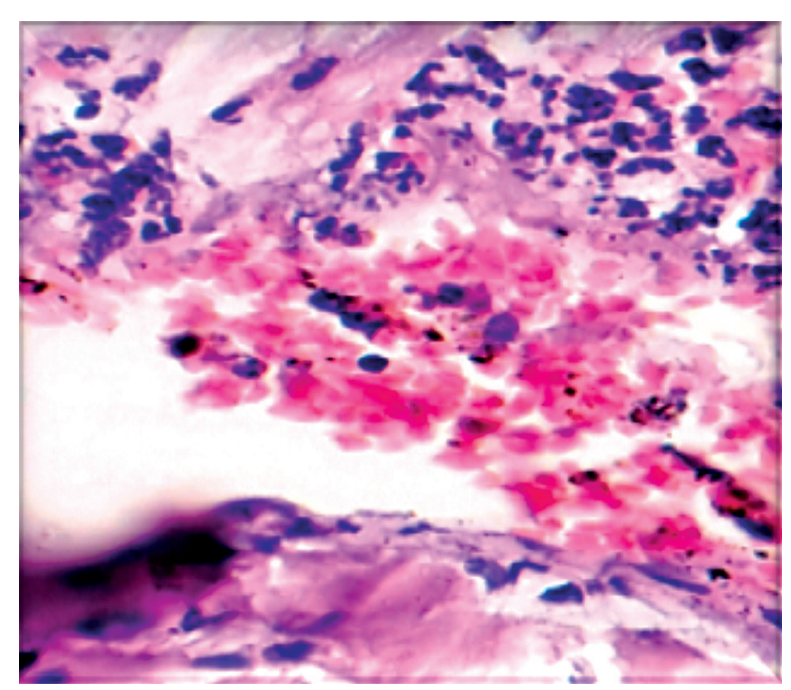

Fig. 13. Section for the cardiac tissue from the valsartan-treated group. The section shows mild cardiac injury. The section was stained with H\&E (40x)

insignificant difference between the control group (II) and control vehicle (III), and the total severity scores of the control vehicle group (III) showed that $14.3 \%$ of the group had moderate cardiac injury, $57.1 \%$ had severe cardiac injury, and $28.5 \%$ had a high severe cardiac injury. Treatment of rats with valsartan improved cardiac injury significantly $(p<0.001)$ compared with the control vehicle (III) group, and the total severity scores of this group showed that $14.3 \%$ of the group had no damage (score 0 ), 
$57.1 \%$ had mild cardiac injury (score 1 ), and $28.5 \%$ had moderate cardiac injury (score 2), as shown in Fig. 9.

\section{Discussion}

Ischaemic heart disease is the primary cause of mortality and morbidity in most developed countries [10]. Reperfusion of the ischaemic myocardium is associated with a dramatic inflammatory response leading to TNF- $\alpha$ release, IL-6 induction, and subsequent neutrophil-mediated cytotoxic injury. It has been reported that the inhibition of apoptosis in a rat model of myocardial infarction reduces infarct size and improves functional ability.

\section{Effects of valsartan on pro-inflammatory cytokines (TNF- $\alpha$ and IL-6) and on the anti-inflammatory cytokine (IL-10)}

Pretreatment with valsartan before induction of myocardial ischaemia produced a significant reduction $(p<0.001)$ in the myocardial tissue levels of pro-inflammatory cytokines (TNF- $\alpha$, IL-6), with a significant elevation $(p<0.001)$ in the level of anti-inflammatory cytokine IL-10 compared to the control group and vehicle group, and this could be attributed to an anti-inflammatory action of valsartan.

Valsartan is an ARB-1, angiotensin II type 1 receptor involved in TNF- $\alpha$ production. In the rat, early, short-term ARB-1 limited post-infarct cardiac TNF- $\alpha$ production and diminished myocardial alterations were observed after myocardial infarction. Also, valsartan inhibited TLR-4 and $\mathrm{NF}-\kappa \mathrm{B}$ expressions concomitant with an improvement in myocardial injury, such as smaller infarct size, fewer release of myocardial enzymes, and proinflammatory mediators. These findings suggest that valsartan plays a pivotal role in the protective effects on myocardial I/R injury. This protection mechanism is possibly due to its anti-inflammatory function via TLR-4/NF- $\kappa \mathrm{B}$ signalling pathway [5].

$\mathrm{Wu}$ et al. found that valsartan attenuated the expression of MCP-1, TNF- $\alpha$, IL-6, IL-1 $\beta$, and infiltration of leukocytes and macrophages in injured arteries [11]. The present study is in agreement with Wu et al. Valsartan inhibited translocation of NF- $\kappa \mathrm{B}$ to the nucleus and decreased plasma TNF- $\alpha$ levels [12].

To the best of our knowledge, there is no data available about the effect of valsartan on IL-10 in regional myocardial ischaemia/reperfusion injury.

\section{Effect of valsartan on caspase 3 and BAX}

The present study showed that there was a significant decrease in myocardial level of caspase- 3 and BAX in the valsartan-treated group compared to the active control group. Wu et al. showed that valsartan inhibited apoptosis and reduced caspase- 3 activity [12]. Ning et al. found that the myocardial apoptosis index was reduced significant- ly in a valsartan-treated group. In addation, Bcl-2 protein was significantly increased while the Bax protein was significantly decreased in rabbits that were subjected to 60 minutes of left anterior descending coronary artery occlusion (LADO) and 6 hours of reperfusion [13]. Jung et al. (2007) found that AgII blocker attenuated the activities of caspase-9, caspase-3, and caspase- 8 and the mRNA levels of Fas-L and TNF- $\alpha$ in normotensive rats with intra-cerebral haemorrhage. They speculated that AgII blocker can improve both intrinsic and extrinsic apoptotic pathway activation [14].

\section{Effect of valsartan on cTnT level}

The present study showed that there was a significant decrease in plasma level of cTnt in the valsartan-treated group compared to the active control group, and this reflects the amelioration of cardiac IR injury by valsartan. Bozcali et al. found that pretreatment with valsartan resulted in a significant decrease in the levels of plasma troponin $\mathrm{T}$ in rats undergoing myocardial ischaemia reperfusion [15]. Goette et al. showed that the AT-II blocker was able to reduce cTnT rise in experimental atrial fibrillation [16]. Fogari et al. observed the lesser increase in cTnT in ARB-treated patients during AF recurrence suggesting a greater protective effect of ARB against the arrhythmia-related myocardial injury [17].

\section{Effect of valsartan on MDA and reduced GSH level}

The present study demonstrates that there is a significant decrease in the myocardial level of MDA in the valsartan-treated group compared to the active control group, and there is significant increase in myocardial level of GSH in the valsartan-treated group compared to the active control group. Wu et al. demonstrated that valsartan pretreatment suppressed MDA content and preserved superoxide dismutase (SOD) activity, consistent with reduced NADPH oxidase expression [12]. Tawfik demonstrated that treatment with both pioglitazone and Ag II blocker have the same attenuating effect on ischaemia reperfusion-induced renal damage in diabetic rats. They prevented renal ischaemia reperfusion-induced lipid peroxidation and protected the kidneys from severe attenuation of antioxidant enzyme activity in rats exposed to renal ischaemia reperfusion [18]. Wu et al. showed that rats pretreated with valsartan for two weeks and then subjected to 30 minutes of ischaemia and four hours of reperfusion suppressed MDA content and preserved SOD activity, consistent with reduced NADPH nicotinamide adenine dinucleotide phosphate oxidase expression [12].

\section{Effect of valsartan on heart parenchyma}

Treatment of rats with valsartan improved cardiac injury significantly compared with the control group, and 
the total severity mean scores of this group showed mild cardiac injury. Wu et al. demonstrated that the ratio of ischaemia reperfusion-induced infarct size was significantly reduced in the valsartan-treated group [12]. Yang et al. showed that valsartan caused significantly less severe myocardial injury and inflammatory cell inflltration [19].

\section{Conclusions}

It can be concluded that valsartan modulates myocardial ischaemia reperfusion injury via interfering with inflammatory, oxidative pathways and apoptosis.

\section{The authors declare no conflict of interest.}

\section{References}

1. Müller V, Losonczy G, Heemann U, et al. (2002): Sexual dimorphism in renal ischemia-reperfusion injury in rats: possible role of endothelin. Kidney Int 2002; 62: 1364-1371.

2. Saydam M, Takka S (2007): Bioavailability file: Valsartan. FABAD J Pharm Sci 2007; 32: 185-196.

3. Jung KH, Chu K, Lee ST, et al. (2007): Blockade of AT1 receptor reduces apoptosis, inflammation, and oxidative stress in normotensive rats with intracerebral hemorrhage. J Pharmacol Exp Ther 322: 1051-1058.

4. Navalkar S, Parthasarathy S, Santanam N, Khan BV (2001): Irbesartan, an angiotensin type 1 receptor inhibitor, regulates markers of inflammation in patients with premature atherosclerosis. J Am Coll Cardiol 37: 440-444.

5. Varagic J, Frohlich ED, Susic D, et al. (2008): AT1 receptor antagonism attenuates target organ effects of salt excess in SHRs without affecting pressure. Am J Physiol Heart Circ Physiol 294: H853-858.

6. Jugdutt BI, Menon V (2008): Upregulation of angiotensin II type 2 receptor and limitation of myocardial stunning by angiotensin II type 1 receptor blockers during reperfused myocardial infarction in the rat. J Cardiovasc Pharmacol Ther 8: 217-226.

7. Wiedemann D, Schneeberger S, Friedl P, et al. (2010): The fibrin-derived peptide B beta (15-42) significantly attenuates ischemia-reperfusion injury in a cardiac transplant model. Transplantation 89: 824-829.

8. Zhang M, Xu YJ, Saini HK, et al. (2005): Pentoxifylline attenuates cardiac dysfunction and reduces TNF-alpha level in ischemic-reperfused heart. Am J Physiol Heart Circ Physiol 289: H832-839.

9. Zingarelli B, Salzman AL, Szabó C (1998): Genetic disruption of poly (ADP-ribose) synthetase inhibits the expression of P-selectin and intercellular adhesion molecule-1 in myocardial ischemia/reperfusion injury. Circ Res 83: 85-94.

10. Mathers CD, Loncar D (2006): Projections of global mortality and burden of disease from 2002 to 2030. PLoS Med 3: e442.

11. Wu L, Iwai M, Nakagami H, et al. Roles of angiotensin II type 2 receptor stimulation associated with selective angiotensin II type 1 receptor blockade with valsartan in the improvement of inflammation-induced vascular injury. Circulation 2001; 104: 2716-2721.
12. Wu B, Lin R, Dai R, et al. (2013): Valsartan attenuates oxidative stress and NF-kappaB activation and reduces myocardial apoptosis after ischemia and reperfusion. Eur J Pharmacol 705: $140-147$

13. Ning XF, Zhang WZ, Liu S, et al. (2008): Cardioprotective effect of valsartan on cardiac ischemic-reperfusion injury. Department of cardiology, the affiliated hospital of Qingdao University Medical College, Qingdao 266003, China.

14. Jung KH, Chu K, Lee ST, et al. (2007): Blockade of AT1 receptor reduces apoptosis, inflammation, and oxidative stress in normotensive rats with intracerebral hemorrhage. J Pharmacol Exp Ther 322: 1051-1058.

15. Bozcali E, Dedeoglu DB, Karpuz V, et al. (2012): Cardioprotective effects of zofenopril, enalapril and valsartan against ischaemia/reperfusion injury as well as doxorubicin cardiotoxicity. Acta Cardiol 67: 87-96.

16. Goette A, Bukowska A, Dobrev D, et al. (2009): Acute atrial tachyarrhythmia induces angiotensin II type 1 receptor-mediated oxidative stress and microvascular flow abnormalities in the ventricles. Eur Heart J 30: 1411-1420.

17. Fogari R, Mugellini A, Zoppi A, et al. (2012): Effect of telmisartan and ramipril on atrial fibrillation recurrence and severity in hypertensive patients with metabolic syndrome and recurrent symptomatic paroxysmal and persistent atrial fibrillation. J Cardiovasc Pharmacol Ther 17: 34-43.

18. Tawfik MK (2012): Renoprotective activity of telmisartan versus pioglitazone on ischemia/reperfusion induced renal damage in diabetic rats. Eur Rev Med Pharmacol Sci 16: 600-609.

19. Yang J, Jiang H, Yang J, et al. (2009): Valsartan preconditioning protects against myocardial ischemia-reperfusion injury through TLR4/NF-kappaB signaling pathway. Mol Cell Biochem 330: 39-46. 